Original article

\title{
Knowledge and awareness among physiotherapy students to combat COVID-19: A questionnaire based study
}

\author{
Mandeep Kumar Jangra *, Akanksha Saxena, Praharsh Anurag \\ Maharishi Markandeshwar Institute of Physiotherapy \& Rehabilitation, Maharishi Markandeshwar (Deemed to be University), Mullana, Ambala, Haryana, India
}

\section{A R T I C L E I N F O}

\section{Keywords:}

COVID-19

Awareness

Knowledge

Physiotherapy

\begin{abstract}
A B S T R A C T
Objective: Despite the various preventive guidelines and recommendation for infection control, the COVID-19 is spreading very extensively. So it becomes essential for healthcare professionals to have proper knowledge and awareness of it. The objective of this study was to assess the level of knowledge and Awareness among Physiotherapy students to combat COVID-19.

Methods: A total of 203 participants from MMIPR, MM(DU), Mullana completed a questionnaire based survey on the Knowledge and Awareness among Physiotherapy students to Combat COVID-19. The questionnaire was selfadministrated and distributed through WhatsApp. Convenient sampling method was used for data collection and the distributions of responses were presented as frequencies and percentages.

Results: A total of 203 physiotherapy students participated in this survey (143 females \& 60 males), forming a response rate of $53.4 \%$ (203 participated out of 380). The overall awareness for all the participants was adequate with $73.1 \%$ reporting correct answers. The findings of this study revealed that $93 \%$ of subjects (score more than $60 \%$ ) have good knowledge and awareness to combat COVID-19. However, only 49\% knew the correct name of the virus causing COVID-19 infection, only $41 \%$ of participants were aware about the diagnostic measures, and only $25.6 \%$ were aware of the treatment strategies. $91 \%$ of the respondents uses 'Aarogya Setu' app, while $85 \%$ says agrees that it helps to know more about the social distancing.

Conclusion: We conclude that physiotherapy students of MMIPR, MM(DU), Mullana (Ambala) were well aware about the knowledge on COVID-19 infection and its preventive measures.
\end{abstract}

\section{Introduction}

In the middle of December 2019, a group of patients admitted in China were diagnosed with"pneumonia of an unknown etiology". 1 Later, it was found that they were suffering from a new disease caused by the Human Corona Virus (HCoV); which was later named as SARS-CoV2. ${ }^{2}$ The first case of Covid-19 was reported in India on January 30 , 2020. After nearly two months COVID-19 spread in almost all parts of the country. ${ }^{3}$ According to WHO situation report updated on 30 August 2020 , there were $24,854,140$ cases of COVID-19 and 8, 38,924 deaths globally. ${ }^{4}$

National Health Commission of China declared that SARS-COV-2 was transmitted through respiratory secretions, droplets, contacts, feces and aerosol transmission is possible. Human-to-human transmission was considered as major mode of transmission. ${ }^{5,6}$ Most patients presented with very mild flu-like symptoms while few patients exhibit severe symptoms and deteriorated rapidly leading to ARDS, respiratory failure, multiple organ failure and even deaths. Common clinical features included fever, cough, shortness of breath, sputum production, fatigue, sore throat and headache. Some of the patients may have gastrointestinal symptoms, with diarrhea and vomiting. ${ }^{7}$

The diagnosis of $2019 \mathrm{CoV}$ infection is based on a history of detailed contact and travel, with RT-PCR and confirmed by next-generation sequencing. ${ }^{7,8}$ Till now, there is no specific medication for SARS-CoV2 management while vaccines have being created all over the world, but their effectiveness is still not known..$^{9-11}$ Major part of the treatment, consists of implementation of infection control measures, symptomatic treatment and respiratory support. ${ }^{5,7}$

IPC measures should be initiated as early as a patient reaches the health care center. Standard precautions include hand hygiene, use of

\footnotetext{
* Corresponding author. Cardiopulmonary Physiotherapy Research Lab, Maharishi Markandeshwar Institute of Physiotherapy \& Rehabilitation, Maharishi Markandeshwar (Deemed to be University), Mullana, Ambala, Haryana, 133207, India.

E-mail addresses: mjangra708@gmail.com, mandeep.jangra@mmumullana.org (M.K. Jangra), akankshasaxena623@gmail.com (A. Saxena), praharshanurag@ gmail.com (P. Anurag).
} 
PPE (Personal protective equipment) to avoid direct contact with patients blood, bodily fluids, secretions and cleaning and disinfection of the equipment's. ${ }^{5}$

So, spread of Corona virus infection can be controlled or reduced from spreading/transmission by practicing the following IPC measures ${ }^{5}$ :

- Frequently wash hands with soap and water (for at least $20 \mathrm{~s}$ )

- Sanitize your hands with alcohol-based hand sanitizer

- Avoid close contact with anyone, when you meet anyone maintain physical distance of at least 6 feet

- Avoid touching your eyes, nose or mouth

- Always cover your nose \& mouth with a mask

- Stay home

- Frequently clean \& disinfect the touched objects \& surfaces

- Follow cough etiquettes

As the incubation period of COVID-19 is less i.e. on an average of 5-14 days presenting with a wide range of symptoms and due to its highly contagious nature, it becomes essential for the physiotherapists to have knowledge and awareness about its transmission, prevention \& control for better screening of patients suffering from the same as they are in direct contact with the patients and are also involved in postCOVID rehab. So this study aims to evaluate the knowledge and awareness of COVID-19 among physiotherapy students.

\section{Materials and methods}

A cross sectional online survey was conducted in June 2020 among Physiotherapy students of a Physiotherapy college in North India. The survey was conducted in the form of an online self-administrated questionnaire using Google Forms.

The questionnaire was developed in English after reviewing the available literature and the International guidelines related to COVID 19. It consisted of 28 questions based on the knowledge, measures to prevent the spread of infections and infection control practices related to COVID 19 and two questions were about the use of 'Aarogya Setu app'. The questionnaire was validated by experienced physiotherapy academicians of MMIPR and modifications were made as per their suggestions. A sample size of 385 was calculated by using the formula: $\mathrm{Z} \alpha^{2}(\mathrm{P})$ $(1-P) / d^{2}(Z=1.96, P=0.53 ; d=5 \%)$ where a response rate of $53 \%$ was taken from a previous study. ${ }^{12}$ The questionnaire was then sent to 385 physiotherapy students of Maharishi Markandeshwar Institute of Physiotherapy \& Rehabilitation, Mullana, Ambala (Haryana), India. Informed consent was taken from all the participants along with their demographic details.

The study was approved by the departmental research committee of Maharishi Markandeshwar Institute of Physiotherapy and Rehabilitation (MMIPR), Mullana, Ambala (Haryana), India (MMIPR/DRC-SPC/ BPT/18/41). This study was done under the guidelines of Helsinki Declaration (revised, 2013) and National guidelines for biomedical and health research involving human participants (Indian Council of Medical Research, 2017). Convenient sampling method was used to collect the data and the distributions of responses were expressed as percentages.

\section{Results}

A total of 203 physiotherapy students ( 143 females \& 60 males) participated in the study; forming a response rate of $52.7 \%$. Among the respondents $66 \%$ (133) were UG students, $23 \%$ (47) were Interns and $11 \%$ (23) were PG students (Fig. 1). The overall awareness of all the participants was adequate with $73 \%$ reporting correct answers. Distributions of scores are described in Fig. 2.

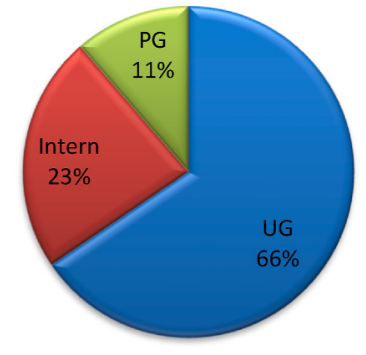

Fig. 1. Qualification of participants.

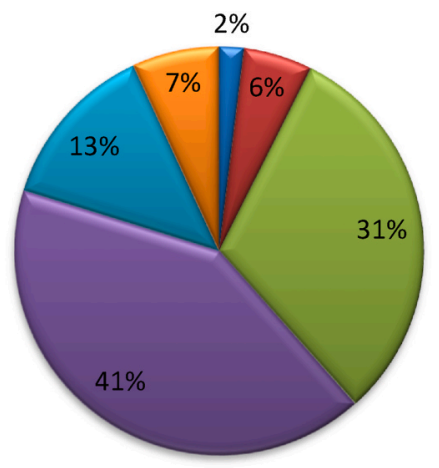

abelow $50 \%$

- $51 \%-60 \%$

$-61 \%-70 \%$

口 $71 \%-80 \%$

- $81 \%-90 \%$

- above $90 \%$

Fig. 2. Distributions of scores.

\subsection{Awareness about COVID-19 infection}

$96 \%$ of the participants were aware that COVID 19 is a viral infection, while only 49\% knew the correct name of virus causing COVID 19. Only $39 \%$ of the participants were aware about the survival of corona virus on the surface of an object. Whereas $60 \%$ of the participants knew that dead body of a COVID-19 positive person can transmit the infection.

$98 \%$ of the participants were well known about the symptoms of COVID-19. Only $86 \%$ participants knew that where the COVID-19 test to be done. Only $41 \%$ of participants knew that both PCR \& NAT test are used to diagnose the COVID- 19 . Only $26 \%$ subjects knew about the treatment of COVID-19 infection. According to $39 \%$ of the participants, there are no specific medications to treat COVID-19 infection, while $52 \%$ says that Hydroxychloroquine is used to treat COVID-19. Only $65 \%$ of the participants were well aware about the national helpline number for COVID-19. Mostly all the participants (91\%) were well aware about the good respiratory hygiene practices (Fig. 3).

\subsection{Awareness about preventive measures}

$98 \%$ of the participants were very well aware about the various measures to prevent the disease from spreading (Fig. 4). Only $68.5 \%$ of the subjects were aware about the duration of hand wash. Only $48.3 \%$ of the subjects were aware about the recommended ethyl alcohol in sanitizer. $58.6 \%$ subjects said that $1 \mathrm{~m}$ is an appropriate distance to maintain social distancing (as per the guideline by the WHO), while $16.7 \%$ said that $2 \mathrm{~m}$ is an appropriate distance to maintain social distancing. $91.1 \%$ of the subjects using the 'AarogyaSetu' app, while $85.2 \%$ said that it helps to know more about the social distancing (Fig. 5).

\section{Discussion}

This survey provided information regarding knowledge and awareness among physiotherapy students to combat COVID-19 at the time of the outbreak in 2020. In this study, 203 physiotherapy students from MMIPR, Mullana (Ambala) were included.

The findings of this study revealed that $93 \%$ of subjects (scored more 


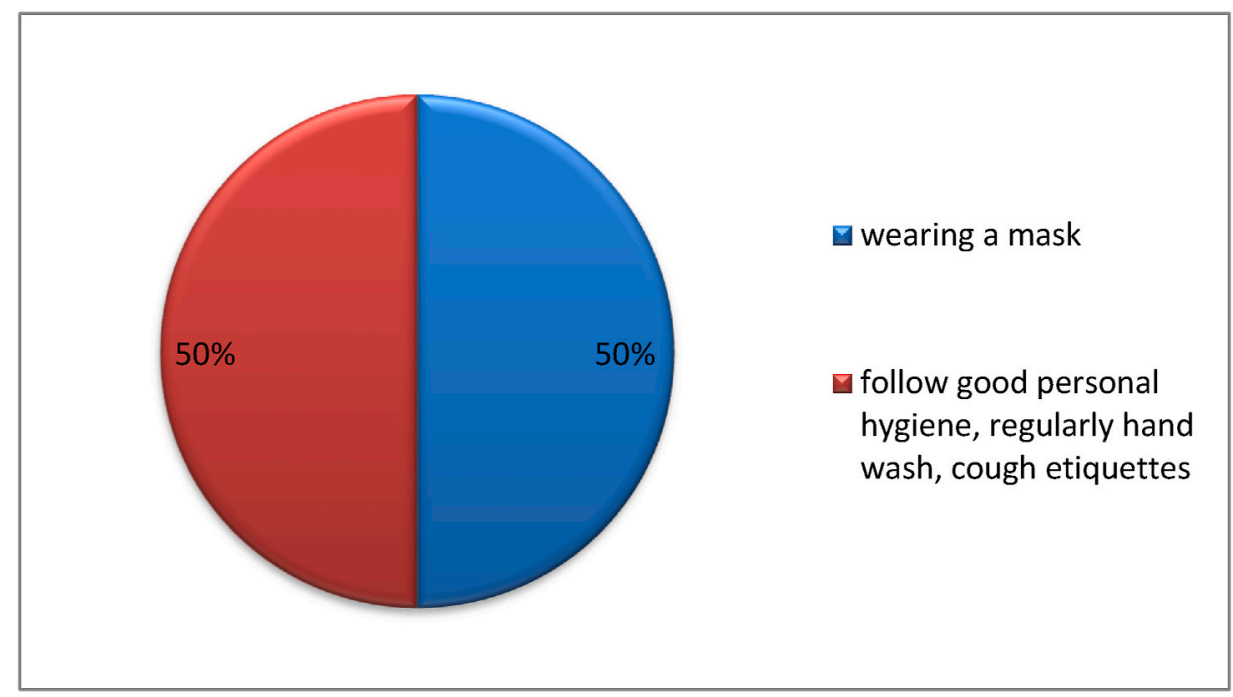

Fig. 3. Awareness about respiratory hygiene practices.

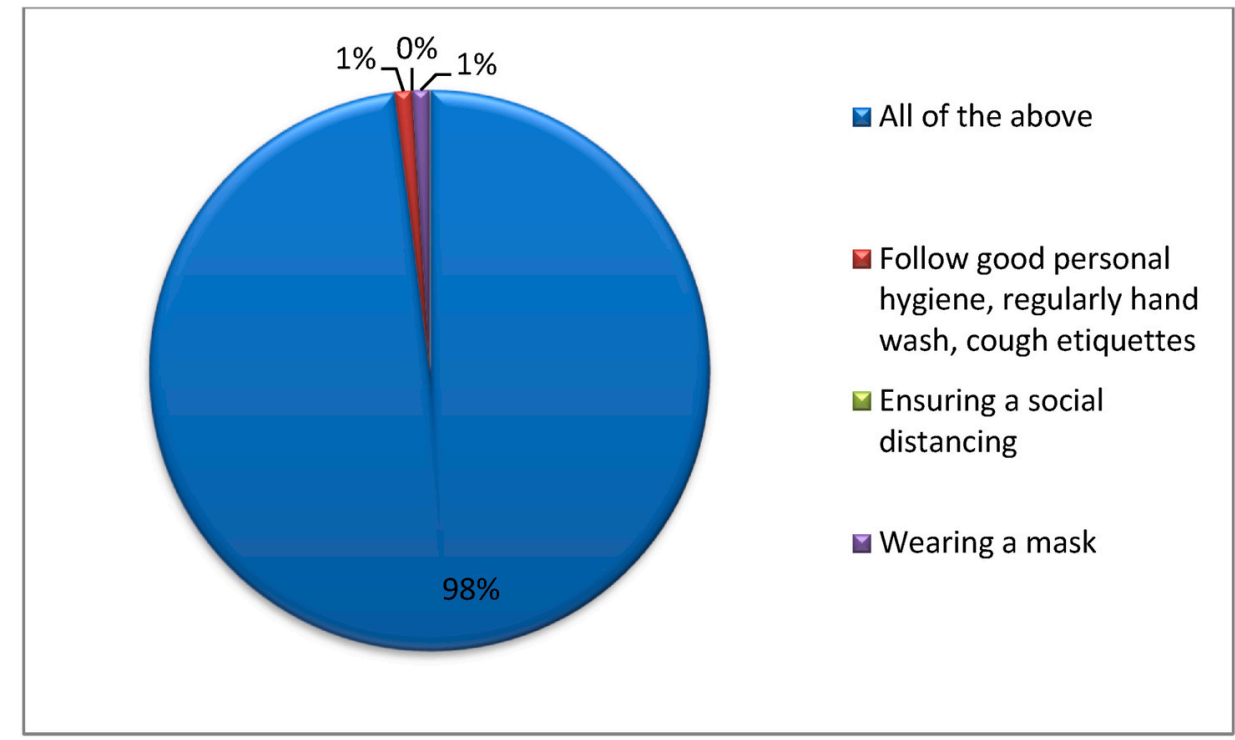

Fig. 4. Awareness about various preventive measures.

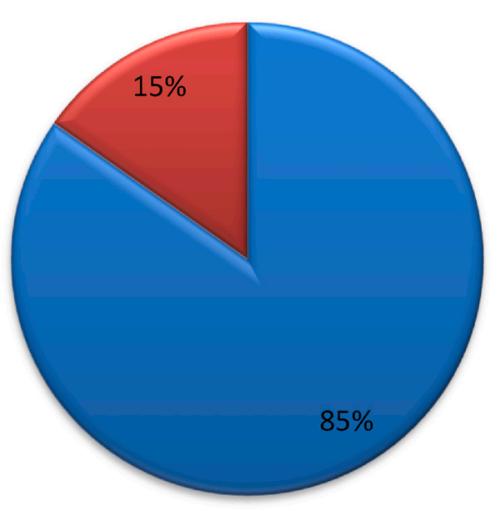

Fig. 5. Usefullness of Aarogya Setu app.

than $60 \%$ ) which depict they have good knowledge and awareness about COVID-19. On the basis of responses to questionnaire we concluded that physiotherapy students (96\%) are well aware that COVID-19 is a viral infection, while only $49 \%$ knew the correct name of the virus that cause COVID-19 infection whereas in the previous study conducted by Pranav D. Modi (2020) to assess COVID-19 awareness among Health care students and professionals in Mumbai metropolitan region, only $22.6 \%$ of the respondents knew the correct name of the causing virus. ${ }^{13}$ This difference in knowledge could be because of the time lapse between the conduct of the two surveys as with time general population got more aware due to the advertisements about the prevention and control of the disease.

In the present study, $91 \%$ of the respondents knew that older persons and individuals with existing comorbidities are at higher risk of getting an infection. Only $9 \%$ respondents say that COVID-19 spreads from pets. Only $60 \%$ respondents are aware that dead body of a COVID- 19 positive person can transmit the infection. Whereas a similar study conducted by Khader et al. to assess the level of awareness regarding the COVID-19 and infection control among Jordanian dentists, majority of the respondents (97\%) were well aware about the symptoms, mode of transmission and measures for preventing COVID-19 transmission. ${ }^{12}$ This difference in the awareness and knowledge could be because majority of the dentists had completed their Post-Graduation and had taken training 
in infection control or attended lectures on COVID-19 prior to the survey.

So according to this physiotherapy students should also follow the same to become more aware regarding diagnostic measures (as only $41 \%$ are aware about this), treatment (only $25.6 \%$ knew about the treatment strategy) and our national helpline no. (as only $65 \%$ respondents knew this) in this pandemic situation. We should improve the knowledge of physiotherapy students on correct causing organism of the COVID-19, laboratory tests to diagnose COVID-19 and treatment strategy to treat COVID-19 by educational programs, webinars and awareness campaigns. On the basis of the above findings, we established that physiotherapy students have good knowledge \& are well aware to combat COVID-19 infection.

This study was the first to assess knowledge \& awareness of COVID19 among physiotherapy students in India. Through this study future researchers can benefit by generalizing how much more awareness is needed in population if healthcare workers are lacking in certain aspects and how to fulfill it. Apart from these, there are certain limitations of this study like could have included different clusters of the healthcare population; there is limited sample size taken from one institute and also results could have been more valid if randomized sample would have been taken.

\section{Conclusion}

Through this e-survey study we conclude that there is sufficient knowledge \& awareness among physiotherapy students of MMIPR, MM (DU), Mullana, Ambala on COVID-19.

\section{Declaration of competing interest}

None.

\section{Appendix A. Supplementary data}

Supplementary data to this article can be found online at https://doi. org/10.1016/j.cegh.2021.100748.

\section{References}

1 Yang X, Yu Y, Xu J, et al. Clinical course and outcomes of critically ill patients with SARS-CoV-2 pneumonia in Wuhan, China: a single-centered, retrospective, observational study. Lancet Respir Med. 2020;8(5):475-481. https://doi.org/ 10.1016/S2213-2600(20)30079-5.

2 Alexander E. Gorbalenya, Susan C. Baker et al. Severe acute respiratory syndromerelated coronavirus: the species and its viruses - a statement of the Coronavirus Study Group. https://doi.org/10.1101/2020.02.07.937862.

3 Arti MK. Modeling and predictions for COVID 19 spread in India. Res Gate. 2020; (April). https://doi.org/10.13140/RG.2.2.11427.81444.

4 https://www.who.int/docs/default-source/coronaviruse/situation-reports /20200831-weekly-epi-update-3.pdf?sfvrsn=d7032a2a_4.

5 Suganthan N. Review Article Covid-19. vol. 31. 2019:3-8, 2.

6 Wang Yixuan, Wang Yuyi, et al. Unique epidemiological and clinical features of the emerging 2019 novel coronavirus pneumonia (COVID-19) implicate special control measures. J Med Virol. 2020;92:568-576. https://doi.org/10.1002/jmv.25748.

7 Yan-Rong Guo, Cao Qing-Dong, et al. The origin, transmission and clinical therapies on coronavirus disease 2019 (COVID-19) outbreak - an update on the status. Military Med Resear. 2020;7(11). https://doi.org/10.1186/s40779-020-00240-0.

8 Sahin AR. 2019 novel coronavirus (COVID-19) outbreak: a review of the current literature. Eurasian J Med Oncol. 2020;4(1):1-7. https://doi.org/10.14744/ ejmo.2020.12220.

9 Rothan Hussin A, Byrareddy Siddappa N. The epidemiology and pathogenesis of coronavirus disease (COVID-19) outbreak. J Autoimmun. 2020;109:102433. https:// doi.org/10.1016/j.jaut.2020.102433.

10 Zhang NN, Li XF, Deng YQ. A thermostable mRNA vaccine against COVID-19. Cell. 2020;182:1271-1283. https://doi.org/10.1016/j.cell.2020.07.024.

11 Le TT, Andreadakis Z, Kumar A. The COVID-19 vaccine development landscape. Nat Rev. 2020;19:305-306. https://doi.org/10.1038/d41573-020-00073-5.

12 Khader Y, Mohannad Nsour Al, et al. Dentists' awareness, perception, and attitude regarding COVID-19 and infection control: cross-sectional study among Jordanian dentists. JMIR Publ Health Surv. 2020;6(2), e18798. https://doi.org/10.2196/18798.

13 Parnav Modi D, Nair Girija, et al. COVID-19 awareness among healthcare students and professionals in Mumbai metropolitan region: a questionnaire- based survey. Cureus. 2020;12(4), e7514. https://doi.org/10.7759/cureus.7514. 